\title{
ORIGINAL
}

\section{ATENCIÓN FARMACÉUTICA EN PERSONAS QUE HAN SUFRIDO EPISODIOS CORONARIOS AGUDOS (ESTUDIO TOMCOR)}

Flor Álvarez de Toledo (1), Pedro Arcos González (2), Teresa Eyaralar Riera (1), Francisco Abal Ferrer F (1), Ana Dago Martínez (1), Laura Cabiedes Miragaya (3), Ignacio Sánchez Posada (4), Gabriela Alvarez Sánchez (1)

(1) Red Española de Atención Primaria.

(2) Departamento de Medicina, Universidad de Oviedo.

(3) Departamento de Economía Aplicada, Universidad de Oviedo.

(4) Hospital Central de Asturias.

\section{RESUMEN}

Fundamento: Este estudio valora los efectos de un nuevo modelo de trabajo en las farmacias, denominado Atención Farmacéutica, frente al modelo tradicional. Se pretende conocer su factibilidad y las diferencias, potencialmente debidas a la Atención Farmacéutica, respecto de los resultados de salud de la farmacoterapia usada, en una muestra de pacientes que han sufrido episodios coronarios agudos.

Métodos: Es un estudio prospectivo con un grupo de intervención (330 personas) y un grupo control (405 personas), realizado en 83 farmacias de Asturias, Barcelona, Madrid y Vizcaya, en las que se hizo seguimiento durante un año del uso de medicamentos en 735 personas, de las cuales finalizaron el estudio 600 .

Resultados: Hubo diferencias favorables al grupo intervención, respecto de: a) uso de servicios sanitarios indicativos de mayor morbilidad, tales como la frecuencia de consultas hospitalarias urgentes por paciente 1,27Interv. (IC95\%:1,10 a 1,44) y 1,63 Contr.(IC95 \%:1,36 a 1,90) o los días promedio de UCI por paciente hospitalizado: 2,46Interv.(IC95\%:1,56 a 3,36) y 5,87 Contr.(IC95 \%:3,57 a 8,17), por causa cardiológica; b) calidad de vida con diferencia de 4,7 $(p<0,05)$ en la dimensión de función física; c) conocimiento de factores de riesgo de enfermedad coronaria, promedio de $+10 \%(p<0,02-0,07$, según dimensión); d) identificación nominal de los medicamentos usados $+10 \%(\mathrm{p}<0,01)$; importancia subjetiva otorgada a los antiagregantes $+12 \%(\mathrm{p}<0,009)$, los beta-bloqueantes, así como sus efectos $+25 \%(\mathrm{p}<0,02)$; y e) satisfacción con la AF y percepción de la competencia profesional, promedio de $+12 \%(\mathfrak{p}<$ $0,000-0,05$, según dimensión).

Conclusiones: Los valores menores de: demanda individual urgente coronaria, frecuencia de hospitalizaciones y número de días de Unidad de Cuidados Intensivos coronaria por hospitalización, sugerirían que los pacientes que tras un episodio coronario agudo reciben Atención Farmacéutica tienden a estar menos enfermos en los doce meses del seguimiento realizado. Bajo el modelo de Atención Farmacéutica los pacientes conocen mejor las razones de su farmacoterapia y, por ello, utilizan mejor los servicios sanitarios del sistema, alcanzando un mayor nivel de salud.

Palabras clave: Atención farmacéutica, Enfermedad coronaria, Servicio farmacéutico

Correspondencia:

Dr. Pedro Arcos González

Departamento de Medicina

Universidad de Oviedo

Campus del Cristo 33006 OVIEDO

Correo electrónico: arcos@correo.uniovi.es

\section{ABSTRACT}

\section{Pharmaceutical Care in People who have had Acute Coronary Episodes (TOMCOR Study)}

Background: This study evaluates the effects on coronary patients of a new practice in community pharmacies called Pharmaceutical Care $(\mathrm{PhC})$ as compared to the traditional pattern of pharmacy practice. It attempts to ascertain whether pharmaceutical care is feasible in addition to ascertaining differences in effectiveness for coronary patients' pharmacotherapeutic health outcomes, potentially attributable to $\mathrm{PhC}$.

Methods: A randomized prospective controlled-intervention study was conducted in 83 community pharmacies in the provinces of Asturias, Barcelona, Madrid and Biscay in a one-year monitoring of the drug-use of 735 patients at the start of the study (330 intervention patients and 405 control) and 600 at the end.

Results: Differences were fund in favor of the intervention group in: a) the use of health care services as a morbidity indicator such as frequency of hospital emergency room visits 1.27 I (CI95 \%; $1.10-1.44)$ and $1.63 \mathrm{C}(\mathrm{CI} 95 \% ; 1.36-1.90)$ or average length-of-stay in Intensive Care Units 2.46 I (CI95\%; $1.56-3.36)$ and $5.87 \mathrm{C}(\mathrm{CI} 95 \% ; 3.57-8.17)$, both due to coronary causes; $b$ ) health-related quality of life score (physical functioning dimension difference of $4.7(\mathrm{p}<0.05)$; $\mathrm{c})$ average patient knowledge of coronary heart disease risk factors having improved by $10 \%(\mathrm{p}<0002-0.007$ depending on dimension); d) patient knowledge of the name and identification of their drugs having improved by $10 \%(\mathrm{p}<0.001)$ along with their subjective perception of the antiagregans drugs relative importance having improved by $12 \%(\mathrm{p}<0.009)$ and effects of beta-blockers having improved by $25 \%(\mathrm{p}<0.02)$; e) average satisfaction with pharmaceutical care service and perception of pharmacist's professional competence having improved by $2 \%$ ( $p<0.000$ to 0.05 depending on dimension).

Conclusions: A decrease in emergency health care demand due to coronary causes, a fewer number of patient hospitalizations and a shorter length-of-stay in Coronary Intensive Care Units due to hospitalization regarding coronary patients on pharmaceutical care would suggest that patients who suffered an acute coronary heart episode made a better use of drugs and would tend to be less ill. Furthermore, coronary patients who received pharmaceutical care services showed a better knowledge of the reasons for their pharmacotherapy and therefore took better advantage of health care resources and improved their health condition.

Key words: Pharmaceutical care. Coronary disease. Pharmaceutical Services. 


\section{INTRODUCCIÓN}

En 1990 los profesores norteamericanos Hepler y Strand definieron la Atención Farmacéutica (AF) como «la provisión responsable de farmacoterapia, con el propósito de conseguir unos determinados resultados que mejoren la calidad de vida del paciente ${ }^{1}$. A partir de 1993, este nuevo concepto aplicado a la práctica farmacéutica se extendió fuera de EEUU y, de hecho, tanto la Organización Mundial de la Salud ${ }^{2}$ como la Federación Internacional Farmacéutica ${ }^{3}$, han publicado sendos documentos en los que consideran el modelo de AF como aquel que permitiría desarrollar mejor las funciones sanitarias hoy exigidas de los farmacéuticos.

En Europa ha habido un amplio acuerdo ${ }^{4,5}$ para utilizar como definición de referencia la antes reseñada y en España el término Pharmaceutical Care ha sido traducido habitualmente desde 1995 como «Atención Farmacéutica». Diferentes iniciativas de investigación, docentes y legislativas, han contribuido a despertar un inusitado interés por la AF en nuestro país desde diferentes perspectivas, a las que no han sido ajenos los intereses corporativos (tanto médicos como farmacéuticos) y las críticas, más basadas en opiniones que en resultados concretos de investigación ${ }^{6}$.

Nuestro grupo entiende que la AF realizada por los farmacéuticos proveedores de medicamentos es una actividad preventiva de primer orden, ya que la vigilancia y seguimiento del uso de los medicamentos en los pacientes no institucionalizados permite detectar precozmente problemas sanitarios que podrían ser graves si no son atajados inicialmente. Una vez valorados y confirmados esos problemas, el farmacéutico puede intervenir por sí mismo o derivar el problema a otro profesional para conseguir su resolución o prevención.

Estos problemas sanitarios, denominados en el contexto de la AF Problemas Relacionados con Medicamentos (PRM) tienen que ver con fallos de efectividad, aparición de reacciones adversas no tolerables, falta de uso de tratamientos indicados o prescritos y uso innecesario de medicamentos ${ }^{7,8}$.

La investigación dirigida a demostrar que la AF mejora los resultados de salud en los pacientes empezó a publicarse en 19989,10. En diciembre de 1999 una nueva revisión ${ }^{11}$ concluyó que era necesario mayor rigor en el diseño, así como medir todas las variables propuestas por Kozma en su modelo $\mathrm{ECHO}^{12}$ (variables capaces de medir resultados Económicos, Clínicos y Humanísticos). En Europa, las primeras publicaciones sobre AF que mostraron mejores resultados en los tres tipos de variables citadas fueron las relativas a los proyectos TOM-Asthma ${ }^{5} \mathrm{y}$ Proyecto $O M A^{13}$ en su parte correspondiente a pacientes cardiacos.

Además de la importancia epidemiológica, otras razones para elegir el grupo de pacientes con enfermedad coronaria fueron: la existencia de evidencia científica sobre la efectividad de los diferentes fármacos usados para prevenir nuevos episodios de la enfermedad o el avance de sus secuelas ${ }^{14-17}$, la contraindicación de muchos medicamentos de no prescripción en pacientes con historia de la enfermedad ${ }^{18-19}$ y el desconocimiento de los resultados del uso inadecuado de los tratamientos farmacológicos cuando son usados principalmente con fines preventivos.

El objetivo general de TOMCOR (Therapeutics Outcomes Monitoring on Coronary Patients) fue evaluar los efectos de la AF en una muestra de farmacias españolas, sobre los pacientes que habían sufrido algún episodio coronario agudo y bajo el modelo de organización sanitaria y farmacéutica de nuestro país. Los objetivos específicos eran:(a) demostrar la factibilidad de la AF en nuestro medio; es decir, si es factible realizar una actividad sistemática y documentada orientada a detectar y resolver Problemas Relacionados con Medicamentos de los pacientes atendidos en las oficinas de farmacia 
de la muestra, por los farmacéuticos que trabajan en ellas, sin más entrenamiento previo específico que el del proceso a seguir; (b) valorar la efectividad de la AF sobre los resultados de salud de la farmacoterapia mediante la detección de una mejora de los resultados clínicos (diferencias entre los grupos de pacientes respecto a la mortalidad, el reinfarto o las consultas a demanda y urgentes del paciente, como indicadores de morbilidad); así como de una mejoría en los resultados sanitarios no clínicos, es decir los denominados resultados humanísticos por Kozma ${ }^{12}$, que son: el aumento del conocimiento de los factores de riesgo de la enfermedad coronaria, el aumento y mejora del conocimiento de los medicamentos usados por cada paciente, la variación y/o mejora de su calidad de vida al final del seguimiento y el grado de satisfacción con la AF, así como los aspectos más apreciados de la misma. La hipótesis a probar era que la AF mejoraría los resultados de salud, precisados en este párrafo, de la población que la recibe.

\section{SUJETOS Y MÉTODOS}

La metodología utilizada, diseñada en la Universidad de Florida y probada en otros estudios hechos en Europa desde 1992 ${ }^{5,20}$, corresponde a un estudio de tipo prospectivo de intervención con grupo de intervención y grupo control, siendo seleccionados al azar los individuos que habían de aprticipar en cada grupo.

En el estudio participaron, de forma voluntaria, 83 farmacias de Asturias, Barcelona, Madrid y Vizcaya, en las cuales se hizo el seguimiento de uso de los medicamentos para las 735 personas que cumplían los criterios de inclusión, los cuales prestaron su consentimiento a participar en el estudio. El tamaño necesario de muestra se calculó respecto a la variable indicadora de morbilidad para la que se disponía de información: la tasa de reinfarto $(10 \%$ en los primeros 6 meses tras el primer infarto), bajo la hipótesis de una potencial disminución del $4 \%$, si se usa adecuadamente la medicación preventiva dentro del programa de AF.

La captación de las farmacias se hizo mediante una carta dirigida a 200 de ellas, cuyos farmacéuticos titulares habían participado en investigaciones previas sobre los servicios de la farmacia comunitaria ${ }^{21,22}$. Se pretendió que tanto los farmacéuticos del grupo control como los del grupo intervención tuviesen el mismo nivel de «motivación» respecto al ejercicio de la Atención Farmacéutica.

Los posibles pacientes coronarios fueron identificados en las farmacias mediante el uso de medicamentos-centinela tales como antiagregantes, anticoagulantes, beta-bloqueantes cardioselectivos, hipolipemiantes y nitratos.

Los criterios para emparejar las farmacias fueron: localización geográfica (área sanitaria de ubicación), atención sanitaria recibida por su población (centros de salud que atendían a sus pacientes y hospitales de referencia para hospitalización) y carga de trabajo (volumen de dispensación/número de trabajadores totales y /número de farmacéuticos). La aleatorización de las farmacias se hizo por grupo, una vez emparejadas.

A los pacientes identificados como posibles participantes en los grupos se les solicitó el informe de alta de su último episodio coronario con hospitalización. Fueron excluidos del estudio todos los pacientes que no aceptaron firmar el documento de consentimiento para participar, así como: (a) los pacientes que no aportaron el informe de alta de su último episodio con hospitalización o cuyo informe no confirmaba el diagnóstico presuntivo de episodio coronario agudo, (b) los pacientes que no eran autónomos para su desplazamiento, (c) los pacientes que planificaban ausentarse del entorno de la farmacia más de tres meses durante el año de seguimiento, (d) los pacientes cuyo informe de alta incluía un diagnóstico de diabetes complicada o enfermedad terminal $\mathrm{y}$, (e) los pacientes del grupo de interven- 
ción cuyo médico de Atención Primaria no aceptó explícitamente participar en el seguimiento de $\mathrm{AF}$ en la farmacia.

De acuerdo con la legislación vigente en el momento del estudio, se pidió consentimiento a los pacientes para registrar sus datos de consumo de medicamentos, archivar datos clínicos en las farmacias y realizar informes farmacoterapéuticos para sus médicos, así como para transferir los datos necesarios a la sede del estudio garantizando su anonimato. Aunque el criterio principal de inclusión de pacientes en el estudio era haber tenido un episodio documentado de enfermedad coronaria, la $\mathrm{AF}$ a cada paciente se realizaba sobre toda su farmacoterapia y necesidades farmacológicas de todas sus patologías.

La intervención hecha por farmacéuticos consistió en realizar AF (prevenir, detectar y resolver PRM) según el protocolo de actuaciones y registros diseñado al efecto $^{23-25}$ en el que fueron entrenados todos los farmacéuticos del grupo intervención. El entrenamiento tuvo dos fases: (a) una primera de doce horas de formación sobre fisiopatología de la enfermedad coronaria y su terapia farmacológica que se impartió por igual a los dos grupos (se pretendía que la única diferencia en el servicio recibido por los pacientes se debiese al proceso específico de AF y no al nivel de conocimientos teóricos de los profesionales) y, (b) otra segunda, sólo para el grupo de intervención, consistente en veinte horas de entrenamiento en el proceso de AF propiamente dicho (oferta del servicio al paciente y a su médico de AP, entrevista inicial y evaluación del plan terapéutico, datos a registrar en las sucesivas visitas que permitan la detección e identificación de PRM, modo de realizar las intervenciones farmacéuticas). Los profesionales del grupo intervención realizaron además dos seminarios acerca de la evaluación y resolución de casos reales, con el fin de homologar la intervención dentro del grupo.

En el grupo control los pacientes fueron atendidos siguiendo el modelo habitual de atención en las farmacias de nuestro país, es decir: ofreciendo consejo sanitario ocasional, sin registrar los datos necesarios para identificar problemas en relación con el uso de medicamentos, ni realizar intervenciones para solventarlos. La colaboración con los médicos de los pacientes en ambos grupos se limitó a la práctica habitual en el grupo control, mientras que en el de intervención los médicos de los pacientes conocían y aceptaban que en la farmacia se registrasen los datos sobre uso de medicamentos descritos en el protocolo y se interviniese sobre ellos cuando, a juicio del farmacéutico, podía mejorarse la efectividad o seguridad de cualquier tratamiento; si ello exigía cambios (supresión, inicio o cambio de pautas) en medicamentos de prescripción, por supuesto, la comunicación con el médico era obligada, así como la aceptación del criterio terapéutico.

Las fuentes de información para la recogida de los datos de mortalidad y morbilidad, fueron los registros efectuados por los farmacéuticos, en ambos grupos, a partir de la documentación clínica aportada por el paciente o sus familiares y de entrevistas personales bimensuales, realizadas en los dos grupos. Las diferencias en resultados clínicos se midieron en términos de mortalidad y morbilidad, considerando para esta variable los reinfartos como indicador directo y el uso de servicios médicos de urgencia y número y características de las hospitalizaciones como indicadores indirectos.

Las diferencias en los resultados no clínicos o «humanísticos» se midieron entre grupos al inicio y al final para la calidad de vida y para las otras variables sólo al final. Para medir la calidad de vida se utilizó el cuestionario SF 36 validado en castellano, cuya fiabilidad, validez y sensibilidad para medir estado de salud en poblaciones con enfermedad coronaria han sido recientemente descritas ${ }^{26}$. Al inicio del estudio, se distribuyó el cuestionario a una muestra del $20 \%$ de las personas participantes en ambos grupos, para comprobar la homogeneidad de la calidad de vida en ese momento. La comparación final entre grupos se realizó entre las medias de cada dimensión, así como frente a los valores pobla- 
cionales medios obtenidos en España por sexo y grupo de edad comparable ${ }^{27}$.

Para medir el conocimiento de los factores de riesgo de enfermedad, el conocimiento de los medicamentos y la satisfacción con el servicio prestado en las farmacias se usaron cuestionarios elaborados específicamente para el estudio TOMCOR, los cuales están a disposición de las personas que los soliciten. Los cuestionarios fueron probados previamente en 40 pacientes de farmacias que realizaban el mismo protocolo de $\mathrm{AF}$ pero que no eran participantes en el estudio $\mathrm{y}$, tras este pilotaje, se modificaron algunos items y sus escalas de medida, distribuyéndose al final del estudio entre los 600 pacientes que perduraban.

El análisis estadístico de las variables cuantitativas se realizó mediante tests de comparación de medias y para las variables cualitativas se usó la prueba de chi cuadra- do; En ambos casos consideramos significativos los valores de $p<0,05$. Para analizar la consistencia interna de los cuestionarios se aplicó la alfa de Cronbach.

\section{RESULTADOS}

De los 735 pacientes (405 control y 330 intervención) que iniciaron el seguimiento, $135(18 \%)$ fueron perdidos a lo largo de los 12 meses; siendo 600 los pacientes que finalizaron (333 control y 267 intervención). Estas pérdidas, sin diferencias significativas entre los dos grupos, se debieron en 26 casos $(3,5 \%)$ a muerte, en $63(8,5 \%)$ a baja de las farmacias participantes y en $46(6 \%)$ a una «verdadera» pérdida de pacientes en el seguimiento.

La tabla 1 muestra las características básicas de los pacientes de ambos grupos al inicio del estudio, que eran similares ex-

Tabla 1

Características de los pacientes al inicio del estudio

\begin{tabular}{|c|c|c|c|}
\hline & Pacientes & $\begin{array}{c}\text { Intervención } N=330 \\
n(\%)\end{array}$ & $\begin{array}{c}\text { Control } N=405 \\
n(\%)\end{array}$ \\
\hline \multirow{4}{*}{ Edad } & $<50$ años & $34(10)$ & $29(7)$ \\
\hline & $50-70$ años & $156(47)$ & $181(44)$ \\
\hline & $>70$ años & $140(42)$ & $195(48)$ \\
\hline & Media & 65,4 & 66,8 \\
\hline \multirow{2}{*}{ Sexo } & Hombres & $234(71)$ & $287(71)$ \\
\hline & Mujeres & $96(29)$ & $118(29)$ \\
\hline \multirow{2}{*}{ Tipo de episodio previo } & Infarto de miocardio & $174(53)$ & $214(53)$ \\
\hline & Angor pectoris & $156(47)$ & $191(47)$ \\
\hline \multirow{2}{*}{ Antigüedad del episodio } & Menor de 1 año & $131(39)$ & $134(33) * *$ \\
\hline & Mayor de 1 año & $199(61)$ & $271(67)$ \\
\hline \multirow{4}{*}{ Factores de riesgo presentes } & Índice Masa Corporal $>25$ & $247(74)$ & $314(77)$ \\
\hline & Presión arterial diastólica $>90 \mathrm{~mm}$ & $40 / 321(12) * *$ & $26 / 399(6,5)$ \\
\hline & Presión arterial sistólica $>140 \mathrm{~mm}$ & $133 / 321(41)$ & $148 / 399(37)$ \\
\hline & Colesterol Total $>200 \mathrm{mg} / 100 \mathrm{ml}$ & $76 / 184(55)$ & $155 / 253(61) * *$ \\
\hline \multirow{3}{*}{ Fármacos utilizados } & Beta Bloqueantes & $127(38)$ & $114(28) * *$ \\
\hline & Antiagregantes & $246(74)$ & $303(75)$ \\
\hline & Hipolipemiantes & $135(40)$ & $144(35)$ \\
\hline
\end{tabular}

** $\mathrm{p}<0,05$ 
cepto en las tres variables referentes a la antigüedad del episodio coronario, la Presión Arterial Diastólica mayor de $90 \mathrm{~mm}$ $\mathrm{Hg}$ y Colesterol Total superior a 200 $\mathrm{mg} / 100 \mathrm{ml}$ en los pacientes para los que se dispuso de los valores bioquímicos, dado que no eran el total de la muestra. En cuanto al perfil terapéutico de medicamentos re- lacionados con su enfermedad coronaria, la única diferencia fue para el grupo terapéutico de beta-bloqueantes. La tabla 2 muestra estas mismas características al inicio, de los 600 pacientes que terminaron el estudio.

Tabla 2

Características, al inicio, de los pacientes que terminaron el estudio

\begin{tabular}{|c|c|c|c|}
\hline & Pacientes & $\begin{array}{c}\text { Intervención } \\
N=267 \\
n(\%)\end{array}$ & $\begin{array}{c}\text { Control } \\
N=333 \\
n(\%)\end{array}$ \\
\hline \multirow{4}{*}{ Edad } & $<50$ años & $28(10,5)$ & $26(7,8)$ \\
\hline & $50-70$ años & $139(52)$ & $176(52)$ \\
\hline & $>70$ años & $100(37,5)$ & $131(39)$ \\
\hline & Media & 64,8 & 65,8 \\
\hline \multirow{2}{*}{ Sexo } & Hombres & $188(70,5)$ & $239(71)$ \\
\hline & Mujeres & $79(29,5)$ & $94(29)$ \\
\hline \multirow{2}{*}{ Tipo de episodio previo } & Infarto de miocardio & $141(53)$ & $169(51)$ \\
\hline & Angor pectoris & $126(47)$ & $164(49)$ \\
\hline \multirow{2}{*}{ Antigüedad del episodio } & Menor de 1 año & $92(34,5)$ & $97(29)$ \\
\hline & Mayor de 1 año & $175(65,5)$ & $236(71)$ \\
\hline \multirow{4}{*}{ Factores de riesgo presentes } & Índice Masa Corporal $>25$ & $202(75)$ & $258(77)$ \\
\hline & Presión arterial diastólica $>90 \mathrm{~mm}$ & $35 / 263(13)$ & $22 / 329(6,6)$ \\
\hline & Presión arterial sistólica $>140 \mathrm{~mm}$ & $112 / 263(42)$ & $119 / 329(36)$ \\
\hline & Colesterol Total $>200 \mathrm{mg} / 100 \mathrm{ml}$ & $57 / 111(51)$ & $134 / 202(66)$ \\
\hline \multirow{3}{*}{ Fármacos utilizados } & Beta Bloqueantes & $103(38)$ & $98(29)$ \\
\hline & Antiagregantes & $201(75)$ & $247(74)$ \\
\hline & Hipolipemiantes & $111(41)$ & $124(37)$ \\
\hline
\end{tabular}

Durante los 12 meses de seguimiento, fallecieron 13 pacientes $(3,2 \%) 4$ de ellos por causa cardiovascular, en el grupo control. Entre los pacientes de intervención también fallecieron 13 (3,9\%), de los cuales, 4 también fueron por causa cardiovascular. Ningún paciente reinfartó dos o más veces a lo largo del seguimiento. La incidencia acumulada de reinfartos en el grupo intervención fue de 14, (4,2\%) mientras que en el grupo control fue de $28(6,9 \%)$ no siendo estadísticamente significativa esta diferencia del $2,7 \%$ en la cifra de reinfartos.
Las diferencias en la utilización de servicios sanitarios (consultas y hospitalizaciones) quedan reflejadas en la tabla 3. Hubo diferencias estadísticamente significativas en cuanto al promedio de visitas por paciente a los servicios de urgencia hospitalarios (1,27 intervención frente a 1,63 control) y el promedio de días de UCI por paciente hospitalizado (2,46 intervención frente a 5,87 control), siempre ambas a causa de enfermedad cardiológica. El número de pacientes hospitalizados por causa cardiológica fue un $2,7 \%$ superior en el grupo intervención, sin 
Tabla 3

Utilización de servicios sanitarios en el Estudio TOMCOR

\begin{tabular}{|c|c|c|c|c|}
\hline \multicolumn{3}{|c|}{ Utilización de servicios } & $\begin{array}{c}\text { Intervención } \\
(N=330)\end{array}$ & $\begin{array}{l}\text { Control } \\
(N=405)\end{array}$ \\
\hline \multirow{6}{*}{$\begin{array}{l}\text { CONSULTAS } \\
\text { N (consultas/paciente) }\end{array}$} & \multirow{3}{*}{ Ordinaria } & Atención Primaria & $4951(15)$ & $5051(12,5)$ \\
\hline & & Especialista Cardiología & $390(2,04)$ & $518(2,04)$ \\
\hline & & Otros especialistas & $334(2,37)$ & $616(3,31)$ \\
\hline & \multirow{3}{*}{ Urgente } & Atención Primaria & $130(1,78)$ & $88(1,66)$ \\
\hline & & $\begin{array}{l}\text { A hospital por } \\
\text { coronarias }\end{array}$ & $\begin{array}{r}70(1,27) * \\
\text { IC95\%: } 1,1-1,44\end{array}$ & $\begin{array}{r}88(1,63) * \\
\text { IC95\%: } 1,36-1,90\end{array}$ \\
\hline & & A hospital por otras causas & $60(1,30)$ & $98(1,42)$ \\
\hline \multirow{3}{*}{$\begin{array}{l}\text { N. }{ }^{0} \text { de pacientes hospitali- } \\
\text { zados n ( } \% \text { del grupo) }\end{array}$} & \multirow{3}{*}{\multicolumn{2}{|c|}{$\begin{array}{l}\text { Por causa cardiológica } \\
\text { En UCI coronaria } \\
\text { Por otras causas }\end{array}$}} & $48(14,5)$ & $48(11,8)$ \\
\hline & & & $13(3,93)$ & $24(5,92)$ \\
\hline & & & $41(12,4)$ & $40(9,97)$ \\
\hline \multirow{6}{*}{$\begin{array}{l}\text { Ingresos hospitalarios } \\
\text { (en días) }\end{array}$} & \multicolumn{2}{|l|}{ Totales } & 1003 & 1183 \\
\hline & \multicolumn{2}{|c|}{ En servicios cardiología } & 477 & 516 \\
\hline & \multicolumn{2}{|c|}{ En otros servicios } & 435 & 523 \\
\hline & \multicolumn{2}{|c|}{ En UCI coronaria } & 32 & 141 \\
\hline & \multicolumn{2}{|c|}{$\begin{array}{l}\text { En UCI coronaria por } \\
\text { paciente hospitalizado }\end{array}$} & $\begin{array}{c}2,46 * \\
(\mathrm{IC} 95 \%: 1,56-3,36)\end{array}$ & $\begin{array}{c}5,87 * \\
(\mathrm{IC} 95 \%: 3,57-8,17)\end{array}$ \\
\hline & \multicolumn{2}{|c|}{ En UCI coronaria por hospitalización } & 0,53 & 1,98 \\
\hline \multirow{2}{*}{$\begin{array}{l}\text { Hospitalizaciones } \\
\text { n(hospitaliz/paciente) }\end{array}$} & \multirow{2}{*}{\multicolumn{2}{|c|}{$\begin{array}{l}\text { Por causa coronaria } \\
\text { Por otras causas }\end{array}$}} & $60(1,25)$ & $71(1,48)$ \\
\hline & & & $49(1,19)$ & $56(1,40)$ \\
\hline
\end{tabular}

** diferencia estadísticamente significativa $(\mathrm{p}<0,05)$

- a excepción de la consulta ordinaria de Atención Primaria que fue utilizada por todos los pacientes de ambos grupos, el resto de los servicios fueron utilizados por diferente número de pacientes en cada uno de ellos

significación estadística, aunque la frecuencia de hospitalización por paciente fue menor en el grupo intervención $(1,25$ frente a $1,48)$.

La tabla 4 muestra los resultados de la calidad de vida. El cuestionario fue distribuido a los 600 pacientes que finalizaron el seguimiento (267 del grupo de intervención y 330 en el grupo control). De ellos, contestaron $184(68,9 \%)$ pacientes de intervención y $242(73,3 \%)$ de control. De las ocho dimensiones generales del cuestionario, el grupo de intervención presentó medias más altas que el control para las cinco dimensiones correspondientes a: función física, papel subjetivo de la función física, dolor físico, salud general y vitalidad, aunque solo la correspondiente a la función física fue estadísticamente significativa $(\mathrm{p}<0,05)$, acercán- dose a los valores normales para su grupo de edad y sexo.

Se recuperaron el 71,1\% de los cuestionarios sobre conocimiento de los factores de riesgo (tabla 5). Se evidenciaron diferencias estadísticamente significativas, a favor del grupo intervención, en cuanto al conocimiento de que el tabaco $(p=0,02)$, la hipertensión $(\mathrm{p}=0,07)$ y la obesidad $(\mathrm{p}=0,03)$ son factores de riesgo para esta enfermedad.

En los otros tres cuestionarios la consistencia interna alcanzó valores entre 0,60 para la dimensión de competencia profesional en el de satisfacción y 0,64 en el de conocimiento de medicamentos.

La recuperación de los cuestionarios sobre conocimiento de medicamentos fue en 
Tabla 4

Diferencias en dimensiones de calidad de vida del Cuestionario SF-36

\begin{tabular}{|c|c|c|c|c|c|c|}
\hline \multirow[b]{2}{*}{ Dimensión de Calidad de vida } & \multicolumn{2}{|c|}{ Intervención } & \multicolumn{2}{|c|}{ Control } & \multirow[b]{2}{*}{ Diferencias } & \multirow{2}{*}{$\begin{array}{c}\text { Población ge } \\
\text { neral: } \\
\text { Varón/Mujer } \\
\text { Misma edad }\end{array}$} \\
\hline & $N$ & $\begin{array}{c}\text { media } \\
(+-D e)\end{array}$ & $N$ & $\begin{array}{c}\text { media } \\
(+-D e)\end{array}$ & & \\
\hline Función física & 179 & $\begin{array}{c}\mathbf{6 4 , 1} \\
(+-23,9)\end{array}$ & 235 & $\begin{array}{c}\mathbf{5 9 , 3} \\
(+-25,3)\end{array}$ & $+4,7 * *$ & $68,9 / 61,3$ \\
\hline Rol físico & 171 & $\begin{array}{c}\mathbf{6 4 , 1} \\
(+-40,0)\end{array}$ & 224 & $\begin{array}{c}\mathbf{6 3 , 0} \\
(+-40,3)\end{array}$ & $+1,1$ & $75,5 / 63,2$ \\
\hline Dolor corporal & 184 & $\begin{array}{c}\mathbf{6 2 , 1} \\
(+-29,7)\end{array}$ & 241 & $\begin{array}{c}\mathbf{6 1 , 3} \\
(+-26,8)\end{array}$ & $+0,8$ & $76,7 / 59,0$ \\
\hline Salud general & 173 & $\begin{array}{c}\mathbf{4 5 , 6} \\
(+-22,2)\end{array}$ & 227 & $\begin{array}{c}\mathbf{4 5 , 4} \\
(+-21,8)\end{array}$ & $+0,2$ & $57,5 / 48,6$ \\
\hline Vitalidad & 175 & $\begin{array}{c}\mathbf{5 3 , 3} \\
(+-23,9)\end{array}$ & 225 & $\begin{array}{c}\mathbf{5 1 , 5} \\
(+-23,5)\end{array}$ & $+1,8$ & $61,3 / 53,1$ \\
\hline Función social & 183 & $\begin{array}{c}\mathbf{7 6 , 7} \\
(+-25,8)\end{array}$ & 242 & $\begin{array}{c}\mathbf{7 8 , 6} \\
(+-25,1)\end{array}$ & $-1,9$ & $86,2 / 79,1$ \\
\hline Rol emocional & 171 & $\begin{array}{c}\mathbf{7 8 , 4} \\
(+-38,0)\end{array}$ & 218 & $\begin{array}{c}\mathbf{7 9 , 3} \\
(+-36,5)\end{array}$ & $-0,9$ & $87,0 / 73,2$ \\
\hline Salud mental & 172 & $\begin{array}{c}\mathbf{6 5 , 3} \\
(+-21,3)\end{array}$ & 222 & $\begin{array}{c}\mathbf{6 7 , 2} \\
(+-21,7)\end{array}$ & $-1,9$ & $75,3 / 63,5$ \\
\hline
\end{tabular}

** $\mathrm{p}<0,05$

$\mathrm{n}$ varía para cada dimensión según la recuperación de cuestionarios

Tabla 5

Diferencias en desconocimiento de factores de riesgo

\begin{tabular}{|lcc|}
\hline \multicolumn{1}{|c|}{ Factores de riesgo } & $\begin{array}{c}\text { Intervención } \\
(N=189) \\
n(\%)\end{array}$ & $\begin{array}{c}\text { Control } \\
(N=238) \\
n(\%)\end{array}$ \\
\hline Tabaco & $12(6,3)$ & $38(15,9) * *$ \\
Hipertensión arterial & $21(11,1)$ & $42(17,6) * *$ \\
Obesidad & $13(6,9)$ & $33(13,9) * *$ \\
Diabetes Mellitus & $103(54)$ & $128(53)$ \\
Estrés & $13(6,8)$ & $22(9,2)$ \\
Dieta lipídica & $10(5,2)$ & $14(5,8)$ \\
Ejercicio & $12(6,3)$ & $15(6,3)$ \\
\hline
\end{tabular}

$\mathrm{P}<0,05$

total del $74,8 \%$. Los pacientes usaban un promedio de 4,5 medicamentos en el grupo de intervención y 4 en el de control (rango de 1 a 9 en ambos grupos). En el grupo de intervención hubo una diferencia estadísticamente significativa $(p=0,017)$ en cuanto a la identificación nominal del medicamento: $129(66,4 \%)$ de los pacientes de interven- ción recordaban el nombre comercial de todos los medicamentos utilizados, frente a $143(56 \%)$ en el grupo control. Tal como se muestra en la tabla 6 , la importancia subjetiva otorgada a los antiagregantes y los beta-bloqueantes así como el conocimiento de su efecto en el contexto de la enfermedad coronaria, tuvieron diferencias significati-

Rev Esp Salud Pública 2001, Vol. 75, N. ${ }^{\circ} 4$ 
Tabla 6

Conocimiento sobre los medicamentos por los pacientes

\begin{tabular}{|c|c|c|c|c|c|c|c|c|}
\hline \multirow{2}{*}{$\begin{array}{c}\text { Grupos } \\
\text { Mediciones }\end{array}$} & \multirow[b]{2}{*}{$N$} & \multicolumn{3}{|c|}{ INTERVENCIÓN } & \multirow[b]{2}{*}{$N$} & \multicolumn{3}{|c|}{ CONTROL } \\
\hline & & $\begin{array}{c}\text { Importancia } \\
\text { Tratamiento } \\
\%\end{array}$ & $\begin{array}{c}\text { Efecto } \\
\text { conocido } \\
\%\end{array}$ & $\begin{array}{c}\text { Duración } \\
\text { Tratamiento } \\
\%\end{array}$ & & $\begin{array}{c}\text { Importancia } \\
\text { Tratamiento } \\
\%\end{array}$ & $\begin{array}{c}\text { Efecto } \\
\text { Conocido } \\
\%\end{array}$ & $\begin{array}{c}\text { Duración } \\
\text { Tratamiento } \\
\%\end{array}$ \\
\hline Hipolipemiantes & 109 & $71,5 \%$ & $87 \%$ & $82,5 \%$ & 123 & $80,4 \%$ & $81,3 \%$ & $76,4 \%$ \\
\hline Beta-bloqueantes & 71 & $78,8 \%$ & $81,6 \% * *$ & $87,3 \%$ & 66 & $80,0 \%$ & $57,5 \% * *$ & $81,8 \%$ \\
\hline Antiagregantes & 153 & $86,9 \% * *$ & $67,3 \%$ & $84,3 \%$ & 186 & $75,8 \% * *$ & $60,2 \%$ & $77,4 \%$ \\
\hline
\end{tabular}

a es diferente para Intervención y Control, en cada grupo terapéutico. Hipolipemiantes: $\mathrm{N}=109$ I N=123 C Beta-bloqueantes: $\mathrm{N}=71 \mathrm{I} \mathrm{N}=66 \mathrm{C}$ Antiagregantes: $\mathrm{N}=153$ I N=186 C ** $\mathrm{p}<0,05$

vas $(\mathrm{p}=0,009$ y $\mathrm{p}=0,002)$ a favor de los pacientes del grupo intervención.

En la tabla 7 se muestran los resultados de satisfacción con la AF entre el 73,1\% de pacientes que contestaron. Los porcentajes medios de satisfacción general y de accesibilidad, así como de relación interpersonal fueron del 79,8\% en el grupo de pacientes de intervención y del 78,2\% en el grupo de pacientes controles. Sí hubo diferencias estadísticamente significativas en la percepción de la competencia profesional a favor del grupo de intervención y también en los porcentajes de satisfacción con el servicio especial de AF

Tabla 7

Diferencias en satisfacción y en aceptación

\begin{tabular}{|c|c|c|}
\hline & $\begin{array}{c}\text { Intervención } N=191 \\
n(\%)\end{array}$ & $\begin{array}{c}\text { Control } N=248 \\
n(\%)\end{array}$ \\
\hline S1 (SGS) & $190(99)$ & $246(99)$ \\
\hline S2 (AF) & $184(96) * *$ & $205(82)$ \\
\hline S3 (AF) & $60(31) * *$ & $56(22,5)$ \\
\hline S4 (ARI) & $168(87,9) * *$ & $185(74,5)$ \\
\hline S5 (SGS) & $122(64)$ & $165(67)$ \\
\hline S6 (ARI) & $151(79)$ & $201(81)$ \\
\hline S7 (CP) & $189(98,9) * *$ & 209 (84) \\
\hline S8 (AF) & $128(67) * *$ & $134(54)$ \\
\hline S9 (CP) & $182(95,2)$ & $202(81,4)$ \\
\hline S10 (ARI) & $159(83)$ & $212(85)$ \\
\hline S11 (SGS) & $168(88)$ & $219(88)$ \\
\hline S12 (ARI) & $95(50)$ & $133(54)$ \\
\hline S13 (AF) & $73(38) * *$ & $71(28,6)$ \\
\hline S14 (ARI) & $184(96)$ & $237(96)$ \\
\hline S15 (AF) & $106(55)$ & $122(49)$ \\
\hline S16 (AF) & $109(57) * *$ & $91(37)$ \\
\hline
\end{tabular}

AF, Servicio de Atención Farmacéutica;

ARI, Accesibilidad y Relación Interpersonal;

CP, Competencia Profesional;

SGS, Satisfacción general con el servicio.

** $\mathrm{p}<0,05$, 


\section{DISCUSIÓN}

Un primer problema planteado en el estudio fue la falta de homologación de las intervenciones de los farmacéuticos del grupo de intervención frente a un mismo problema relacionado con un medicamento. Esta cuestión puede plantear dudas a la hora de asignar determinados resultados al proceso de AF. Por otro lado, esta es una dificultad bastante habitual cuando lo que se trata de probar es un nuevo modelo de servicio sanitario que aún no está completamente definido.

La totalidad de resultados citados por Kozma incluye los económicos que, en nuestro caso, serán objeto de una publicación diferenciada y completan el estudio de efectividad.

Los resultados relativos a los indicadores de morbilidad (menor tasa de reinfarto, menor demanda individual de atención urgente por causa coronaria, menor número de hospitalizaciones por paciente y menor número de días de UCI coronaria por hospitalización) indican que aquellos pacientes que, habiendo sufrido un episodio coronario agudo y recibido una atención especial sobre su modo de usar los medicamentos (AF), parecen estar menos enfermos durante los doce meses del seguimiento realizado.

Como la AF no es una intervención sanitaria directa sobre la patología, sino indirecta sobre uno de los recursos terapéuticos (los medicamentos) del que ya se ha probado la eficacia, lo que verdaderamente probarían nuestros resultados es que la efectividad de los medicamentos para cada sujeto depende en gran parte del uso correcto, entendiendo por «uso correcto» no sólo la utilización adecuada en cuanto a cumplimiento de la posología y correcta forma de administración sino el valor que asigna a cada medicamento el propio enfermo, lo que podríamos llamar educación sanitaria sobre medicamentos. En los dos grupos de nuestro estudio se daban por igual la información al prescribir (mismas consultas médicas y hos- pitales) y la información farmacéutica al dispensar, (misma formación farmacológica en los farmacéuticos de ambos grupos), pero el grado de conocimiento alcanzado para la enfermedad y su tratamiento fue superior en los pacientes que recibieron AF.

Trabajos similares al nuestro se han realizado en grupos de enfermos con otras patologías cardiacas no coronarias ${ }^{13,28}$ obteniéndose igualmente una disminución del número de hospitalizaciones o de consultas en los servicios de urgencia. También se ha experimentado con pacientes asmáticos en los que los resultados esperados de la comprensión de la enfermedad y su terapéutica disminuyen los días de absentismo laboral o escolar en población joven y adolescente ${ }^{20}$.

Las diferencias en las cifras de reinfarto, el mayor y más claro indicador de morbilidad, aunque no son estadísticamente significativas, son coherentes con el número de consultas urgentes, el rango y media de los días de hospitalización por paciente y la utilización de UCI coronaria una vez hospitalizados. Una posible razón para explicar la paradoja de estas cifras en relación con el mayor porcentaje de pacientes que son hospitalizados en el grupo de intervención, sería que los pacientes aprendieron que la asistencia sanitaria para la enfermedad coronaria debe ser prestada con urgencia en el medio hospitalario y, por tanto, un mayor número de pacientes de ese grupo utilizó ese servicio. Así mismo, mejoró la utilización de los medicamentos y ambas situaciones condujeron a que, aún siendo mayor el porcentaje de población que usó el hospital, en cada ingreso la gravedad fue menor y el número de ingresos por paciente que estuvo enfermo fue muy inferior. Dada la importancia de lo observado (disminución de la tasa de reinfarto) y su ausencia de significación estadística, será interesante probar este hallazgo en estudios con muestras más grandes.

Este mejor estado de salud de los pacientes atendidos con AF se correlaciona bien, a 
su vez, con los resultados obtenidos para las otras variables con las que medimos los resultados sanitarios no clínicos en ambos grupos. No obstante, al haber utilizado cuestionarios no validados que hubo que desarrollar «ad hoc»y pilotar en pacientes de farmacias no participantes, subsisten las limitaciones habituales en estos casos. Por otro lado, pueden existir reservas acerca de la atribución a la AF de algunas de las diferencias en estos resultados no clínicos, debido a la ausencia de una aplicación inicial de los cuestionarios.

Es especialmente llamativo el mejor conocimiento de que el tabaco, la hipertensión y la obesidad están relacionados con la enfermedad coronaria, ya que según el estudio Framingham, en su última edición ${ }^{29}$, éstos siguen siendo importantes en la prevención secundaria. El conocimiento de los medicamentos señala claras diferencias respecto a la memorización del nombre y el valor terapéutico asignado por los pacientes a los beta-bloqueantes y antiagregantes cuya utilidad en el control de la prevención secundaria de esta enfermedad ha vuelto a ser puesta de manifiesto recientemente ${ }^{30}$.

Los resultados de medir la satisfacción con el nuevo servicio demuestran que, aún siendo alta la satisfacción con el servicio recibido en las farmacias, los usuarios que recibían AF apreciaban el plus de calidad sanitaria que se les ofertaba.

En conclusión: Los pacientes atendidos en farmacias en las que se utilizó el modelo de AF tuvieron una mejor calidad de vida en lo referente a la dimensión de función física del cuestionario SF36. Estos pacientes conocían mejor que sus controles que el tabaco, la hipertensión y la obesidad eran importantes factores de riesgo para su enfermedad, memorizaban mejor el nombre del medicamento que usaban y daban más importancia para el control de su enfermedad a los antiagregantes y beta-bloqueantes. Esto significa que el modelo de AF desarrollado hace que los pacientes conozcan mejor las razones de su farmacoterapia y, en consecuencia, utilicen mejor los servicios sanitarios que el sistema pone a su disposición, alcanzando un mayor nivel de salud.

\section{AGRADECIMIENTOS}

A la Red Española de Atención Primaria, sociedad científica promotora de la investigación. Al Area de Medicina Preventiva y Salud Pública de la Universidad de Oviedo que fue sede material de la investigación durante los 3 años en que se realizó. A MSDEspaña que financió el estudio y a los Colegios Oficiales de Farmacéuticos de Barcelona y Vizcaya que respaldaron y colaboraron económicamente en el mismo. A los médicos de familia/generales que aceptaron la nueva forma de trabajar de los farmacéuticos con los pacientes en el grupo intervención, a los pacientes y a los farmacéuticos comunitarios capaces de innovar su práctica profesional farmacéutica. También agradecemos: el asesoramiento técnico del Dr. Hepler de la Universidad de Florida; la coordinación de los farmacéuticos y las farmacias: en Barcelona por Benet Fité Novellas, en Madrid por Horacio del Barrio Sánchez y en Vizcaya por Joseba Ruiz Golvano. A Raimundo Pastor Sánchez (secretario de la REAP) e Iñigo Gorostiza Hormaetxe (becario en la Universidad de Florida al inicio del estudio), por su participación en la revisión final del manuscrito.

\section{BIBLIOGRAFÍA}

1. Hepler CD, Strand LM, Opportunities and Responsabilities in Pharmaceutical Care. Am J Hosp Pharm 1990; 47:533-543.

2. World Health Organization. Second Meeting on the Role of the Pharmacist in the Health Care System. (Q.H.O./PHARM/94.569); 1993 Aug 31 - Sept 1; Tokio: WHO 1993.

3. The International Pharmaceutical Federation. The Tokyo Declaration. Standards for quality of Pharmacy Services (Good Pharmacy Practice). Amsterdam: FIP 1993. 
4. Kielgast PJ. Pharmaceutical Care. Int J Pharm Practice 1993; 5:125-126.

5. Van Mil JWF. Pharmaceutical Care, the future of Pharmacy. Theory, Research and Practice. Doctoral Dissertation. RIJKSUNIVERSITEIT GRONINGEN. 2000 january 28. Druk; Drukkerij De Volharding: Groningen, 1999.

6. Costas Lombardía E. Análisis crítico de la Atención Farmacéutica. Medicina General 2000; 25:591-598.

7. Strand LM. Drug related problems: their structure and function.Ann Pharmacother 1990; 24:10931097.

8. Panel de consenso «ad hoc». Consenso de Granada sobre Problemas relacionados con medicamentos, Pharm Care Esp 1999; 1:107-112.

9. Jack E. Fincham. Pharmaceutical Care studies: a review and update. Drug Benefit Trends 1998; $41-45$

10. Kennie Natalie R, Schuster Brenda G, Einarson Thomas R. Critical Analysis of the Pharmaceutical Care Research Literature. Ann Pharmacother 1998; 32:17-26.

11. Singhal Puneet K, Raisch Dennis W, Gupchup Gireesh V. The impact of pharmaceutical services in community and ambulatory Care Settings: evidence and recommendations for future research. Ann Pharmacother 1999; 33:1336-1355.

12. Kozma CM, Reeder CE, Schultz RM. Economic, clinical and humanistic outcomes: a planning model for pharmacoeconomic research. Clinical Therapeutics 1993; 15(6):1121-1132.

13. Varma S, McElnay JC, Hughes CM, Passmore AP, Varma M. Pharmaceutical Care of patients with cardiac heart failure: interventions and outcomes. Pharmacotherapy 1999; 19(7):860-869.

14. BHAT (Beta-blocker Heart Attack Trial) Research Group. A randomized trial of propanolol in patients with acute myocardial infarction.1. Mortality results. JAMA 1982; 247:1707-1714.

15. ATC (Antiplatelet Trialists' Collaboration): collaborative overview of randomized trials of antiplatelet therapy I: prevention of death, myocardial infarction and stroke by prolonged antiplatelet therapy in various categories of patients. BMJ 1994; 308:81.
16. Randomized trial of cholesterol lowering in 4444 patients with coronary heart disease: The scandinavian simvastatin survival study (4S). Lancet 1994; 44:1383-1390.

17. Sacks FM et al.The effect of pravastatin on coronary events after myocardial infarction in patient with average cholesterol levels. N Eng J Med 1997; 335:1001-1008

18. Gascón Lecha M.P. Atención Farmacéutica y Oficina de farmacia. El Farmacéutico 1998; (200 supl):12-14.

19. Alexander A. Counter prescribing for patients at risk. (5) Cardiac patients. The pharmaceutical Journal 1992 Feb 15; pg. 223-224.

20. Herborg H, Sondergaard B, Frokjaer B, Fonnesbaek L. Quality improvement of drug therapy for asthma patients: a development project and controlled trial in danish community pharmacies. In Hepler CD (ed). Pharmaceutical Care and community Practice. Jerusalem: FIP, 1996; pg. 1-12.

21. Álvarez de Toledo F, Zardaín E, Eyaralar T, Dago AM, Arcos P. Detección del error de prescripción en la dispensación farmacéutica: incidencia y tipología. Aten Primaria 1993; 11(2):70-74

22. Álvarez de Toledo F, Arcos González P, Cabiedes Miragaya L, Dago Martínez A, Eyaralar Riera T. Costes y efectividad sanitaria de la intervención farmacéutica en Oficinas de Farmacia. Farmacoeconomía 1995, II, 9-20

23. Eyaralar Riera MT, Álvarez de Toledo Saavedra F, Dago Martinez AM, Pastor Sanchez R, Alvarez Sánchez G, Arcos González P. Actividades del proceso de Atención Farmacéutica: la visita farmacéutica. Pharm Care Esp 1999; 1:70-75.

24. Eyaralar Riera MT, Álvarez de Toledo Saavedra F, Dago Martínez AM, Pastor Sánchez R, Álvarez Sánchez G, Arcos González P. Actividades del proceso de Atención Farmacéutica: la entrevista inicial con el paciente. Pharm Care Esp 1999 1:145-151

25. Cabiedes Miragaya L, Eyaralar Riera MT. Evaluación económica de la Atención Farmacéutica: una aproximación metodológica. Pharm Care Esp 1999;1:369-381.

26. Dempster M, Donnelly M. Measuring the health related quality of life of people with ischaemic heart disease. Heart 2000; 83(6):641-644.

Rev Esp Salud Pública 2001, Vol. 75, N. ${ }^{\circ} 4$ 
ATENCIÓN FARMACÉUTICA EN PERSONAS QUE HAN SUFRIDO EPISODIOS CORONARIOS AGUDOS...

27. Alonso J, Regidor E, Barrio G, Prieto L, Rodríguez $\mathrm{C}$, de la Fuente L. Valores poblacionales de referencia de la versión española del cuestionario de salud SF36. Med Clin (Barc) 1998; 111:410416.

28. Gattis WA, Hasselblad V, Whellan DJ, O'Connor CM. Reduction in Heart Failure Assessment Recomendation and Monitoring (PHARM) Study. Arch Intern Med 1999; 159:1939-1945.
29. D'Agostino RB, Russell MW, et al. Primary and subsequent coronary risk appraisal: New results from the Framingham Study. Am Heart J 2000; 139:272-281.

30. Gottlieb SS, McCarter RJ, Vogel RA. Effect of beta-blockade on mortality among high-risk and low-risk patients after myocardial infarction. $\mathrm{N}$ Engl J Med 1998; 339:489-497. 